\title{
Supporting Information for \\ Formaldehyde Encapsulated in Zeolite: A Long-lived, Highly Activated One-carbon Electrophile to Carbonyl-ene Reactions
}

Takahiro Okachi and Makoto Onaka*

Department of Chemistry, Graduate School of Arts and Sciences, The University of Tokyo, Komaba, Meguro, Tokyo 153-8902, Japan.

\section{General Experimental:}

Infrared (IR) spectra were recorded on a JASCO FT/IR-550 Fourier transform infrared spectrometer. ${ }^{1} \mathrm{H}$ NMR spectra were recorded on a JNM-GSX $270(270 \mathrm{MHz}) .{ }^{13} \mathrm{C}$ NMR spectra were recorded on a JNM-GSX $270(68 \mathrm{MHz}) .{ }^{13} \mathrm{C}$ MAS NMR spectra were recorded at $25{ }^{\circ} \mathrm{C}$ on a CMX-300 (75 MHz). High-resolution mass spectra (HRMS) were recorded on a JEOL JMS-700V GCmate. Analytical TLC was performed on Merck DC-Platten Kieselgel 60 F254 plates. Silica gel column chromatography was carried out with Merck Kieselgel (60-mesh). NaX (UNION SHOWA Co., LTD., 13X, Si/Al=1.5) and NaY (TOSOH Co., LTD., 320NAA, Si/Al=2.7) were purchased in a powder form, and activated at $400{ }^{\circ} \mathrm{C}$ under vacuum for 4 hours. Paraformaldehyde was purchased from Tokyo Kasei Kogyo Co., LTD. Paraformaldehyde $\left(99 \%{ }^{13} \mathrm{C}\right.$-enriched) was purchased from Cambridge Isotopes. Commercial samples of alkenes, hexane, cyclohexane, and methanol were purchased and used as received. Styrenes were prepared from the corresponding acetophenones and methyltriphenylphosphonium bromide by the Wittig reaction.

\footnotetext{
${ }^{13}$ C MAS NMR Spectra.

${ }^{13} \mathrm{C}$ NMR spectra were recorded at $25{ }^{\circ} \mathrm{C}$ using the single-pulse sequences with ${ }^{1} \mathrm{H}$ high power decoupling under MAS conditions at a spinning rate of $4.5 \mathrm{KHz}$.
}

\section{Preparation of HCHO@NaY or NaX.}

The gaseous formaldehyde was generated by the thermal depolymerization of paraformaldehyde $(4.0 \mathrm{~g}) \quad$ at $150{ }^{\circ} \mathrm{C}$ and induced to the flask containing $\mathrm{NaY}$ or $\mathrm{NaX}(30.0 \mathrm{~g})$ at $0{ }^{\circ} \mathrm{C}$. Maximum adsorption of $\mathrm{HCHO}$ was observed with equivalent units of $\mathrm{HCHO}$ one half that of sodium cations in the zeolite (about $72 \mathrm{mg}$ of $\mathrm{HCHO}$ per $\mathrm{g}$ of $\mathrm{NaX}$ or NaY, ca. $2.4 \mathrm{mmol} / \mathrm{g})$.

\section{Typical experimental procedure for the carbonyl-ene reaction using HCHO@NaY.}

To a solution of $3^{\prime}, 4^{\prime}$-dichloro- $\alpha$-methylstyrene $(1.0 \mathrm{mmol})$ in cyclohexane $(4 \mathrm{ml})$ was added HCHO@ NaY (3.0 mmol as $\mathrm{HCHO})$ at $20{ }^{\circ} \mathrm{C}$. After the mixture was stirred at $20{ }^{\circ} \mathrm{C}$ for $1 \mathrm{~h}, \mathrm{MeOH}(5 \mathrm{ml})$ was added. The mixture was stirred for $10 \mathrm{~min}$, and then the NaY was filtered out, washed with methanol three times. The filtrate was evaporated under reduced pressure. The crude products were chromatographed on silica gel to give 3-(3',4'-dichlorophenyl)-3-buten-1-ol in 99\% yield.

\section{3-(3',4'-Dichlorophenyl)-3-buten-1-ol:}

IR $\left.\left(\mathrm{CCl}_{4}\right) 3628,3393,3087,2951,2881,1626,1549,1473,1384,682 \mathrm{~cm}^{-1} .{ }^{1} \mathrm{H} \mathrm{NMR}_{\left(\mathrm{CDCl}_{3}\right)}\right) \delta 1.55(\mathrm{~s}, 1 \mathrm{H}), 2.72(\mathrm{dt}$, $J=1.1$ and $6.5 \mathrm{~Hz}, 2 \mathrm{H}), 3.73(\mathrm{t}, J=6.5 \mathrm{~Hz}, 2 \mathrm{H}), 5.21(\mathrm{~d}, J=1.1 \mathrm{~Hz}, 1 \mathrm{H}), 5.41(\mathrm{~d}, J=1.1 \mathrm{~Hz}, 1 \mathrm{H}), 7.24(\mathrm{dd}, J=2.2 \mathrm{and}$ $8.6 \mathrm{~Hz}, 1 \mathrm{H}), 7.40(\mathrm{~d}, J=8.6 \mathrm{~Hz}, 1 \mathrm{H}), 7.49(\mathrm{~d}, J=2.4 \mathrm{~Hz}, 1 \mathrm{H}) .{ }^{13} \mathrm{C} \mathrm{NMR}\left(\mathrm{CDCl}_{3}\right): \delta 38.2,60.8,115.9,125.3,128.0$, 
130.2, 131.4, 132.4, 140.5, 142.7. HRMS: Calcd for $\mathrm{C}_{10} \mathrm{H}_{10} \mathrm{OCl}_{2}\left(\mathrm{M}^{+}\right)$216.0109. found 216.0103. Anal: Calcd for $\mathrm{C}_{10} \mathrm{H}_{10} \mathrm{OCl}_{2}$ : C, 55.33; H, 4.64; Cl, 32.66. found: C, 55.18; H, 4.62; Cl, 32.74.

\section{3-Phenyl-3-buten-1-ol: ${ }^{1}$}

IR $\left(\mathrm{CCl}_{4}\right)$ 3628, 3365, 3084, 3059, 3026, 2948, 2880, 1627, 1574, 1495, 1445, $705 \mathrm{~cm}^{-1} .{ }^{1} \mathrm{H} \mathrm{NMR}\left(\mathrm{CDCl}_{3}\right) \delta 1.47(\mathrm{~s}$, $1 \mathrm{H}), 2.79(\mathrm{dt}, J=1.4$ and $6.5 \mathrm{~Hz}, 2 \mathrm{H}), 3.73(\mathrm{t}, J=6.5 \mathrm{~Hz}, 2 \mathrm{H}), 5.16(\mathrm{~d}, J=1.4 \mathrm{~Hz}, 1 \mathrm{H}), 5.41(\mathrm{~d}, J=1.4 \mathrm{~Hz}, 1 \mathrm{H})$,

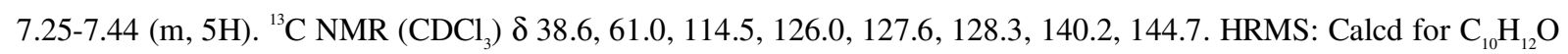
$\left(\mathrm{M}^{+}\right)$148.0888. found 148.0887. Anal: Calcd for $\mathrm{C}_{10} \mathrm{H}_{12} \mathrm{O}: \mathrm{C}, 81.04 ; \mathrm{H}, 8.16$. found: C, 80.91; H, 8.36.

\section{2-Butyl-3-methyl-3-buten-1-ol:}

IR $\left(\mathrm{CCl}_{4}\right) 3294,3157,2943,2851,1630,1470 \mathrm{~cm}^{-1} .{ }^{1} \mathrm{H} \mathrm{NMR}\left(\mathrm{CDCl}_{3}\right) \delta 0.89(\mathrm{t}, J=0.7 \mathrm{~Hz}, 3 \mathrm{H}), 1.20-1.36(\mathrm{~m}, 6 \mathrm{H})$, $1.67(\mathrm{~s}, 3 \mathrm{H}), 2.24-2.30(\mathrm{~m}, 1 \mathrm{H}), 3.45-3.59(\mathrm{~m}, 2 \mathrm{H}), 4.91(\mathrm{~d}, J=5.5 \mathrm{~Hz}, 2 \mathrm{H}) .{ }^{13} \mathrm{C} \mathrm{NMR}\left(\mathrm{CDCl}_{3}\right)$ 14.0, 18.5, 22.7, 28.9, 29.4, 50.0, 64.0, 113.7, 145.4. HRMS: Calcd for $\mathrm{C}_{9} \mathrm{H}_{18} \mathrm{O}\left(\mathrm{M}^{+}\right)$142.1358. found 142.1351.

Ene reaction with 1-methylcyclohexene. ${ }^{2}$ The isomeric ratio of three ene products were determined to be $50: 50$ by ${ }^{1} \mathrm{H}$ NMR.

\section{2-Mehtylenecyclohexylmethanol:}

${ }^{1} \mathrm{H}$ NMR $\left(\mathrm{CDCl}_{3}\right) \delta$ 1.20-2.40 (m, 8H), 3.57-3.60 (m, 1H), 3.76-3.80 (m, 1H), $4.72(\mathrm{~d}, J=6.0 \mathrm{~Hz}, 2 \mathrm{H})$.

\section{2-Mehtylcyclohex-2-enylmethanol:}

${ }^{1} \mathrm{H} \mathrm{NMR}\left(\mathrm{CDCl}_{3}\right) \delta 1.50-2.00(\mathrm{~m}, 4 \mathrm{H}), 1.70(\mathrm{~s}, 3 \mathrm{H}), 1.98(\mathrm{~m}, 2 \mathrm{H}), 2.24(\mathrm{~m}, 1 \mathrm{H}), 3.63-3.70(\mathrm{~m}, 2 \mathrm{H}), 5.58(\mathrm{~m}, 1 \mathrm{H})$.

Ene reaction with Limonene. ${ }^{2}$ The isomeric ratio of three ene products were determined to be 20:13:67:0 by ${ }^{1} \mathrm{H}$ NMR. 5-Isopropenyl-2-methylcyclohex-2-enylmethanol:

${ }^{1} \mathrm{H}$ NMR $\left.\left(\mathrm{CDCl}_{3}\right) \delta 1.37-2.32(\mathrm{~m}, 7 \mathrm{H}), 1.66(\mathrm{~s}, 3 \mathrm{H}), 1.70 \mathrm{~s}, 3 \mathrm{H}\right), 3.6-3.8(\mathrm{~m}, 2 \mathrm{H}), 4.71(\mathrm{~s}, 2 \mathrm{H}), 5.56(\mathrm{~s}, 1 \mathrm{H})$.

\section{References and Notes}

1. Okachi, T.; Fujimoto, K.; Onaka, M. Org. Lett. 2002,4, 1667.

2. (a) Maruoka, K.; Concepcion, A. B.; Murase, N.; Oishi, M.; Hirayama, N.; Yamamoto, H. J. Am. Chem. Soc. 1993, 115, 3943. (b) Snider, B. B.; Rodini, D. J.; Kirk, C.; Cordova, R. J. Am. Chem. Soc. 1982, 104, 555. 


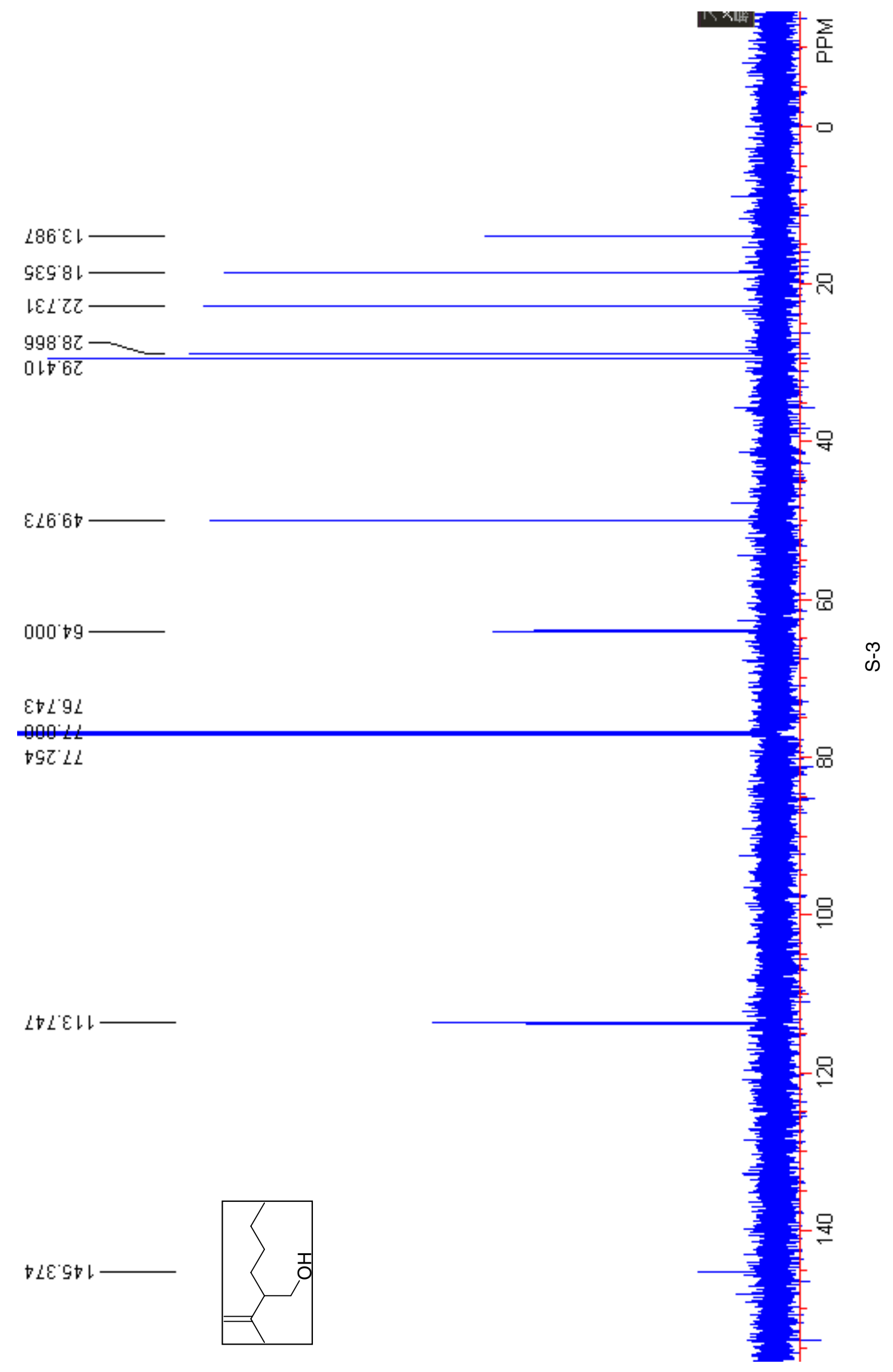




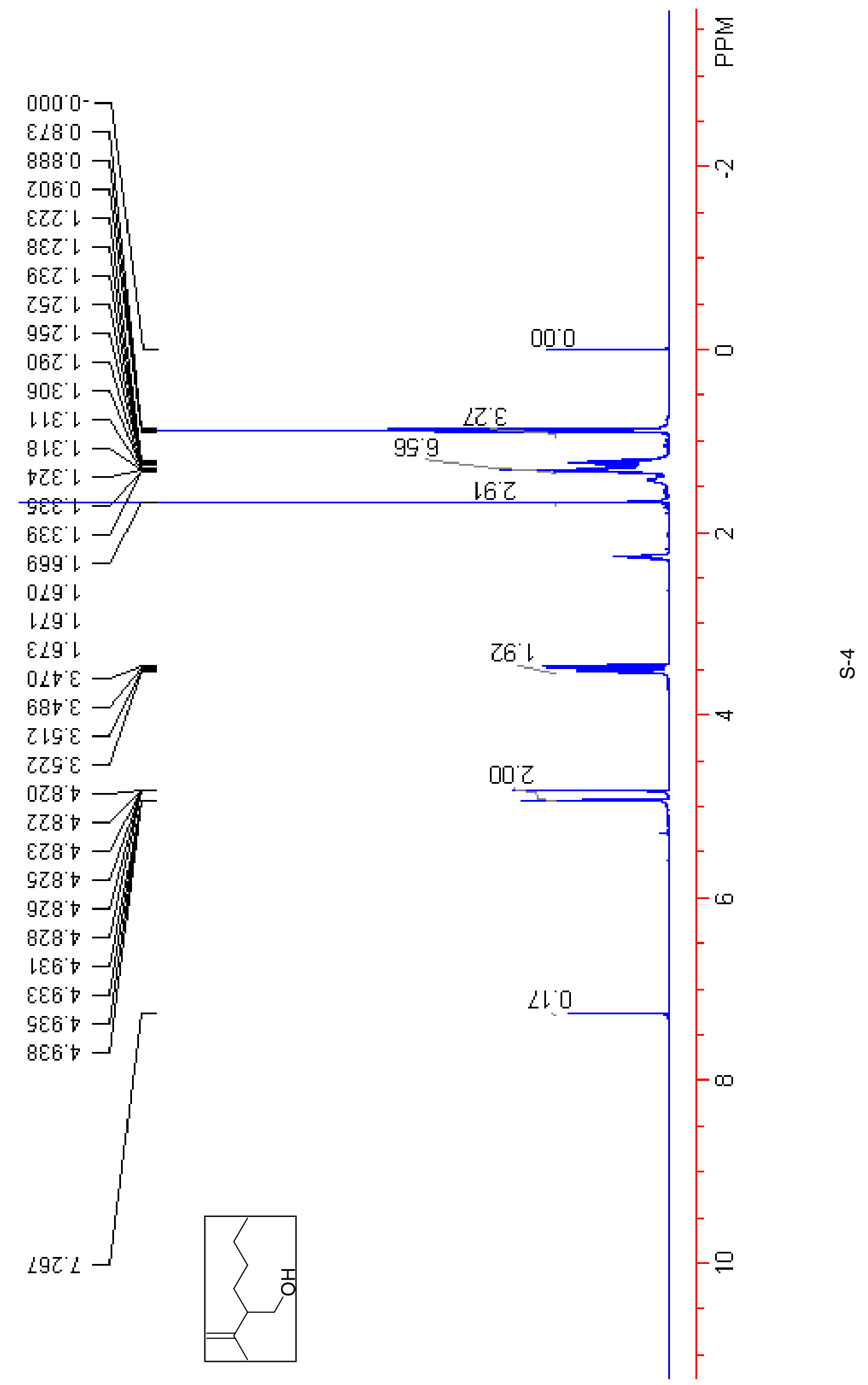

